\title{
Modeled Microgravity Disrupts Collagen I/Integrin Signaling During Osteoblastic Differentiation of Human Mesenchymal Stem Cells
}

Valerie E. Meyers ${ }^{1}$, Majd Zayzafoon ${ }^{1}$, Steven R. Gonda², William E. Gathings ${ }^{3}$, Jay M. McDonald ${ }^{1,4, \#}$

${ }^{1}$ Dept of Pathology, University of Alabama, Birmingham, Birmingham, AL 35294

${ }^{2}$ Cellular Biotechnology Program, NASA JSC, Houston, TX

${ }^{3}$ CMDS, University of Alabama in Huntsville, Huntsville, AL 35899

${ }^{4}$ Veterans Administration Medical Center, Birmingham, AL 35233

\# Correspondence to:

Jay M. McDonald, MD

Department of Pathology

University of Alabama at Birmingham

$70119^{\text {th }}$ Street South

LHRB 519

Birmingham, AL 35294-0007

Phone: 205-934-6666

Fax: 205-975-9927

Email: imcdonald@path.uab.edu

Running title: Integrin Function in Modeled Microgravity

Key Words:

- Osteoblast

- HARV

- RCCS

- Collagen

- FAK

- MAPK

- Integrin

Total \# of Figures: 7

Contract grant sponsor: ; Contract grant number: 


\begin{abstract}
Spaceflight leads to reduced bone mineral density in weight bearing bones that is primarily attributed to a reduction in bone formation. We have previously demonstrated severely reduced osteoblastogenesis of human mesenchymal stem cells (hMSC) following seven days culture in modeled microgravity. One potential mechanism for reduced osteoblastic differentiation is disruption of type I collagen-integrin interactions and reduced integrin signaling. Integrins are heterodimeric transmembrane receptors that bind extracellular matrix proteins and produce signals essential for proper cellular function, survival, and differentiation. Therefore, we investigated the effects of modeled microgravity on integrin expression and function in hMSC. We demonstrate that seven days of culture in modeled microgravity leads to reduced expression of the extracellular matrix protein, type I collagen (Col I). Conversely, modeled microgravity consistently increases Col l-specific $\alpha_{2}$ and $\beta_{1}$ integrin protein expression. Despite this increase in integrin subunit expression, autophosphorylation of adhesion-dependent kinases, focal adhesion kinase (FAK) and proline-rich tyrosine kinase 2 (PYK2), is significantly reduced. Activation of Akt is unaffected by the reduction in FAK activation. However, reduced downstream signaling via the Ras-MAPK pathway is evidenced by a reduction in Ras and ERK activation. Taken together, our findings indicate that modeled microgravity decreases integrin/MAPK signaling, which likely contributes to the observed reduction in osteoblastogenesis.
\end{abstract}


Altered human bone homeostasis during spaceflight results in severe decreases in bone mineral density, the severity of which directly correlates with flight duration [Bikle and Halloran, 1999]. It has been estimated that 1-2\% site-specific bone loss occurs in the human skeleton each month during spaceflight [Tilton et al., 1980]. Investigations of the potential mechanisms underlying this phenomenon will provide insight into the causes of disuse and age-related osteoporosis. Several independent studies of humans during spaceflight have detected decreased serum levels of bone formation markers, including alkaline phosphatase, osteocalcin, and the C-terminal peptide of pro-collagen type I [Caillot-Augusseau et al., 2000; Collet et al., 19971. Previous in vitro studies of osteosarcoma cells in microgravity have also identified a reduction in markers of osteoblast function, including type I collagen, alkaline phosphatase, and osteocalcin gene expression [Carmeliet et al., 1998; Landis et al., 2000].

Due to payload constraints, flight frequency, and cost, spaceflight experiments are limited. Therefore, NASA has developed a commercially available rotary cell culture system (RCCS) to model microgravity in ground-based experiments. We have previously used and described this system in detail [Zayzafoon et al., 2004]. The high aspect ratio vessels (HARVs), used in the RCCS, provide two essential components of optimized suspension culture: 1) Solid body rotation and 2) Diffusion-mediated oxygenation. Solid body rotation of the vessel, media, microcarriers, and cells results in minimal shear stress and mechanical damage to cells. Membrane oxygenation allows diffusion of gases to maintain proper growth conditions but prevents turbulence-inducing air space/bubbles.

Although some disagreement exists regarding the validity of ground-based microgravity experiments, several cell types have been studied using the RCCS, and many of these studies reveal strikingly similar results to those obtained during spaceflight. In addition, this groundbased system provides an opportunity to study, and potentially correct, disruptions in normal cellular physiology. Studies using osteoblastic cells in the RCCS indicate a reduction in osteoblastic gene markers, including alkaline phosphatase, osteocalcin, and Runt-related 
transcription factor 2 (Runx2) [Nakamura et al., 2003; Narayanan et al., 2002]. It is possible that reduced differentiation of precursor cells, in addition to reduced osteoblastic function, contributes to the observed reduction in bone formation during spaceflight. Indeed, despite osteogenic induction, human mesenchymal stem cells (hMSC) failed to display detectable levels of mRNA for major osteoblastic markers, including alkaline phosphatase, procollagen type I, osteonectin, and Runx 2, following 7 days of culture in modeled microgravity [Zayzafoon et al., 2004]. However, the mechanisms underlying reduced differentiation remain unclear.

Extracellular matrix (ECM) proteins and their heterodimeric transmembrane integrin receptors activate signaling cascades that lead, ultimately, to cell survival, proliferation, or differentiation. Ligand specificity of integrin receptors is determined by the subunit composition of the receptor pair [Hynes, 2002]. When integrins bind ECM proteins, they cluster and are activated, leading to their association with cytoskeletal and signaling complexes. Following integrin ligation, adhesion-dependent tyrosine kinases, including focal adhesion kinase (FAK) and proline-rich tyrosine kinase 2 (PYK2), are activated by autophosphorylation [Juliano, 2002]. PYK2 is best characterized in osteoclasts [Xiong and Feng, 2003], however, its function in osteoblasts remains unclear. Activation of PYK2 has been linked to the anabolic action of fluoride [Jeschke et al., 1998], and a recent study demonstrated an increase in PYK2 activation following mechanical stretching of ROS 17/2.8 osteoblastic cells [Boutahar et al., 2004]. Activation of FAK contributes to the survival and differentiation of osteoblastic cells [Takeuchi et al., 1997; Tamura et al., 2001]. Indeed, altered attachment and spreading, in addition to reduced FAK activation, has been described in osteoblasts isolated from patients suffering from osteoporosis or osteoarthritis [Perinpanayagam et al., 2001]. FAK has been implicated in PI3K activation, leading to survival signaling through activation of Akt [Hanks et al., 2003]. Akt activation is reduced in a mouse unloading model [Sakata et al., 2004]. However, the role of $\mathrm{PI3K}$ signaling in osteoblastic differentiation is controversial [Ghosh-Choudhury et al., 2002; Takeuchi et al., 1997; Vinals et al., 2002]. Another potential role for FAK in osteoblastic 
differentiation is through activation of the Ras-MAPK pathway [Suzawa et al., 2002]. This leads to activation of extracellular signal-regulated kinase (ERK), which is essential for osteoblastic differentiation [Lai et al., 2001]. ERK mediates osteoblastic differentiation through its activation of Runx2, an essential osteoblastic transcription factor [Xiao et al., 2000].

Type I collagen is the most abundant protein in the extracellular matrix of bone, and is required for osteoblastic differentiation. It has been shown that type I collagen expression in decreased in hMSC isolated from osteoporosis patients [Rodriguez et al., 2000]. Additionally, blocking either collagen fibril formation or Col I interaction with its integrin receptor, $\alpha_{2} \beta_{1}$, in vitro reduces alkaline phosphatase expression [Takeuchi et al., 1997]. It has also been shown that collagen/integrin signaling cooperates with bone morphogenetic protein (BMP) signaling to fully induce osteoblastic differentiation, and it is likely that these two pathways converge on the MAPK pathway [Xiao et al., 2002].

Here, we demonstrate that expression of Col I is dramatically reduced in hMSC following 7 days of culture in modeled microgravity. Integrin expression and function are likewise altered in modeled microgravity. Despite elevated Col I-specific integrin subunit expression, activation of adhesion-dependent tyrosine kinases, FAK and PYK2, is significantly reduced. Survival signaling through Akt remains intact. However, activation of Ras and MAPK is significantly reduced. 


\section{MATERIALS AND METHODS}

\section{Isolation of Human Mesenchymal Stem Cells}

Human mesenchymal stem cells were isolated by the UAB Bone Cell Production Core Facility, with approval from the Institutional Review Board at the University of Alabama at Birmingham, as previously described [Zayzafoon et al., 2004]. Briefly, cells were flushed from surgical waste ribs or femoral heads and purified by Histopaque-1077 (Sigma, St. Louis, MO) gradient density centrifugation. Stromal cells that remained attached after 7 days of undisturbed culture in T-175 flasks were maintained and fed every 3-4 days thereafter. The pluripotent potential of these cells has been described previously [Pittenger et al., 1999]. All cells used in this study were between passage 5 and 7 . Donors vary in age, race, and gender. However, our results were consistent regardless of the source of the cells.

\section{Cell Culture and Differentiation}

Human mesenchymal stem cells were maintained as described previously [Zayzafoon et al., 2004]. Briefly, $10^{6}$ cells were seeded onto $50 \mathrm{mg}$ polystyrene microcarrier beads (Solohill Engineering Labs, Ann Arbor, MI) in ultra low adhesion tissue culture plates in normal gravity for 7 days in proliferation medium, consisting of DMEM and $10 \%$ FBS. The medium was changed twice during this period. Cells were then transferred to either $10 \mathrm{~mL}$ high aspect ratio vessels (HARVs) in the rotary cell culture system (RCCS) to model microgravity or ultra low adhesion $100 \mathrm{~mm}$ plates for gravity controls. The proliferation medium was supplemented with $10 \mathrm{nM}$ dexamethasone, $10 \mathrm{mM} \beta$-glycerol phosphate, and $50 \mu \mathrm{M}$ ascorbic acid 2-phosphate to induce osteoblastogenesis. The medium was changed every 2 days in both modeled microgravity and normal gravity cell cultures. Cells were harvested 7 days after the initiation of modeled microgravity. 


\section{Rotary Cell Culture System}

The Rotary Cell Culture System (Model HARV, size $10 \mathrm{~mL}$ ) was purchased from Synthecon, Houston, TX. The high aspect ratio vessels (HARVs) used by the system are fitted with a gas permeable membrane to allow passive gas exchange. The system achieves optimized suspension culture conditions by rotational randomization of the gravity vector. During the 7-day exposure to modeled microgravity, rotation was increased as needed to compensate for the increasing mass of cell/bead aggregates [Zayzafoon et al., 2004].

\section{RNA Extraction and RT-PCR}

Total RNA was extracted, using the TRIzol method as recommended by the manufacturer (Invitrogen, Carlsbad, CA). RNA was reverse transcribed and processed for PCR reactions as described previously [Zayzafoon et al., 2004]. TaqMan real-time semi-quantitative RT-PCR analysis was performed using the relative-standard curve method with SYBRGreen on an ABI Prism® 7900HT Sequence Detection System (Applied Biosystems, Foster City, CA). Expression of the 18S rRNA subunit served as a control. The primers used were identical to those described previously [Zayzafoon et al., 2004].

\section{Whole Cell Protein Extraction}

Whole cell protein extraction was carried out as described previously [Zayzafoon et al., 2004]. Briefly, cells were washed with chilled PBS and flash frozen in liquid nitrogen. Cells were then resuspended in lysis buffer containing $50 \mathrm{mM}$ Tris (pH 7.4), $150 \mathrm{mM} \mathrm{NaCl}, 1 \mathrm{mM}$ EDTA, and $1 \%$ Triton X-100. Protease and phosphatase inhibitor cocktails (Sigma, St. Louis, MO) were added to the lysis buffer immediately prior to cell lysis. Cells were lysed for 30 minutes at $4^{\circ} \mathrm{C}$, and samples were centrifuged at $14,000 \times \mathrm{xg}$ for 30 minutes at $4^{\circ} \mathrm{C}$. The protein content of the supernatant was determined by the Bio-Rad DC protein assay. 


\section{Western Blot Analysis}

Whole cell protein extracts $(20 \mu \mathrm{g} / \mathrm{lane})$ were separated by SDS-PAGE and transferred to a polyvinylidene difluoride (PVDF) Immobilon-P membrane (Millipore Co., Billerica, MA) using a Bio-Rad wet transfer system. Protein transfer efficiency and size determination were verified using prestained protein markers (Bio-Rad, Hercules, CA). Membranes were blocked with Blotto B (Santa Cruz Biotechnology, Santa Cruz, CA) for 1 hour at room temperature followed by overnight incubation at $4^{\circ} \mathrm{C}$ with primary antibodies directed against integrin $\beta_{1}$, phosphorylated and total FAK and ERK (Santa Cruz Biotechnology, Santa Cruz, CA), integrin $\alpha_{2}$ (BD Transduction Laboratories, San Diego, CA), and phosphorylated and total Akt (Cell Signaling Technology, Inc., Beverly, MA). Primary antibody binding was detected using an HRP-conjugated secondary antibody and an enhanced chemiluminescence detection system (Amersham Bioscience, Piscataway, NJ).

\section{Ras Activity Assay}

The Ras activity assay was performed according to the manufacturer's recommendations (Upstate Biotechnology, Inc., Waltham, MA). Lysates were collected and adjusted to a total protein concentration of $150 \mu \mathrm{g} / \mathrm{sample}$. Positive and negative controls were established by incubating normal gravity control samples with $0.5 \mathrm{M}$ EDTA and either GTP (1X) or GDP (1X), respectively, for 30 minutes at $32^{\circ} \mathrm{C}$. Activated (GTP-bound) Ras was precipitated with Raf- 1 conjugated agarose beads for 45 minutes at $4^{\circ} \mathrm{C}$. The beads were collected by centrifugation and washed three times with lysis buffer. Activated Ras was then detached from the beads by boiling for 5 minutes in Laemmli reducing buffer (1X) immediately prior to separation by $12.5 \%$ SDS-PAGE and transfer to PVDF membrane. After blocking, the membranes were incubated with a primary antibody directed against Ras overnight at $4^{\circ} \mathrm{C}$. 
Signals were detected using an HRP-conjugated anti-mouse IgG2a secondary antibody and an enhanced chemiluminescence detection system (Amersham Bioscience, Piscataway, NJ).

\section{Hydroxyproline Assay}

To determine collagen matrix deposition, hydroxyproline analysis was performed by the Biomolecular Resource Facility at the University of Texas Medical Branch in Galveston, TX. Briefly, aggregates were hydrolyzed in $6 \mathrm{~N} \mathrm{HCl}$ at $107^{\circ} \mathrm{C}$. Samples were allowed to dry completely and were then reconstituted in $500 \mu \mathrm{L} 0.02 \mathrm{~N} \mathrm{HCl}$. Protein hydrolysates $(10 \mu \mathrm{L})$ were then analyzed by ion exchange chromatography on a Hitachi L-8800 amino acid analyzer as described by the manufacturer after Spackman et. al. [Moore et al., 1958]. Sample concentrations were derived by comparison to an external standard.

\section{Statistical Analysis}

Statistical analyses were performed using the Student $t$-test on pixel intensity data generated from scanning autoradiographs. Significance was accepted at a $P$ value of $<0.05$. All experiments were repeated at least three times. Values are expressed as mean \pm SE. 


\section{RESULTS}

First, we confirmed that modeled microgravity inhibits osteoblastic differentiation of human mesenchymal stem cells (hMSC). HMSC were allowed to form aggregates on polystyrene beads. The aggregates were then cultured under osteogenic conditions in normal gravity or modeled microgravity for 7 days. Semi-quantitative RT-PCR analyses of early markers of osteoblastogenesis, revealed significant reductions in alkaline phosphatase and Runx2 gene expression (figure 1). Reduced gene expression of type I procollagen (Col1 $\alpha 2$ ), an essential extracellular matrix component of bone, was also detected (figure 2). Hydroxyproline analysis confirmed a dramatic reduction in collagen matrix accumulation in samples cultured in modeled microgravity. Average hydroxyproline content in normal gravity was $26.3 \pm 2.9$ nmol/aggregate ( $n=2$ in duplicate), whereas the hydroxyproline content of samples cultured in modeled microgravity was below detectable limits (figure $2 \mathrm{~B}$ ).

It has been shown that Coll 1 and interaction with its specific integrin receptor, $\alpha_{2} \beta_{1}$, are required for osteoblastic differentiation. To examine the effect of modeled microgravity on Col Ispecific integrin receptor expression, we analyzed the protein expression of both $\alpha_{2}$ and $\beta_{1}$ integrin subunits by Western blot. Densitometric analysis revealed a $136 \pm 21 \%$ increase in $\alpha_{2}$ and a $145 \pm 17 \%$ increase in $\beta_{1}$ expression by cells cultured in modeled microgravity relative to gravity controls (figure 3). However, this effect appears to be subunit specific as protein expression of the $\alpha_{5}$ integrin subunit was slightly reduced by $26 \pm 10 \%$ in modeled microgravity (data not shown). Post-transfer coomassie blue protein staining of the polyacrylamide gel was used to ensure loading standardization.

To evaluate integrin function, we examined the activation of adhesion-dependent kinases downstream of integrin binding. Western blot analysis revealed no change in total FAK or PYK2 protein expression between cells cultured in modeled microgravity and cells cultured in 
normal gravity. However, autophosphorylation of FAK and PYK2 was significantly reduced by $65 \pm 11 \%$ and $50 \pm 9 \%$ in cells cultured in modeled microgravity, as determined by densitometry (figure 4). Downstream activation of the small GTP-protein, Ras, was significantly reduced by $79 \pm 10 \%$ under modeled microgravity conditions (figure $5 \mathrm{~A}$ ). Subsequent phosphorylation of the MAPK, ERK, was similarly reduced by $73 \pm 7 \%$. However, activation of cellular survival signaling through AKT was unaffected by modeled microgravity (figure 6). 


\section{DISCUSSION}

Reduced osteoblastogenesis of hMSC following 7 days of modeled microgravity was confirmed by reduced gene expression of two osteoblastic markers, alkaline phosphatase and Runx2. This is consistent with previous data [Nakamura et al., 2003; Zayzafoon et al., 2004]. We further examined the expression of another osteoblastic marker, Col I. Col I is the major extracellular matrix component in bone and is secreted by osteoblasts during differentiation [Owen et al., 1990; Siggelkow et al., 1999]. Alterations in Col I are implicated in postmenopausal osteoporosis, as disruption of collagen fibrils in the bone matrix occurs in mice following ovariectomy [Kafantari et al., 2000]. In vitro, inhibition of collagen synthesis blocks ascorbic acid-induced osteocalcin promoter activity in MC3T3-E1 cells [Xiao et al., 1997]. Additionally, treatment of either the rat osteosarcoma UMR-106-01 cell line or human bone marrow stromal cells with antibodies directed against the collagen integrin receptor, $\alpha_{2} \beta_{1}$, leads to a dramatic reduction in mineralization [Gronthos et al., 2001; Schneider et al., 2001]. This reduction in osteoblastic gene expression and mineralization appears to be mediated by reduced Col I induction of Runx2 binding and OSE2 transcriptional activation [Xiao et al., 1998]. Consistent with reduced osteoblastic differentiation, we detected reduced gene expression of Col1a2 in modeled microgravity. Further confirmation of impaired collagen matrix accumulation is evidenced by the reduction in hydroxyproline levels from aggregates of hMSC cultured in modeled microgravity. Conversely, a previous study using hypergravity, induced by centrifugation, detected an increase rather than a decrease in Col I gene expression [Gebken et al., 1999].

Interestingly, protein expression of the collagen-binding $\alpha_{2}$ and $\beta_{1}$ integrin subunits was increased in modeled microgravity. This is in contrast to a recent report of reduced expression of $\alpha_{v}$ and $\beta_{3}$ integrin subunits after 14 days in a mouse hindlimb suspension model of unloading 
[Sakata et al., 2004]. One possible explanation for the discrepancy is subunit specific regulation, as expression of other integrin subunits, such as $\alpha_{5}$, was slightly reduced in our system (data not shown). Differences in integrin trafficking may provide an additional explanation for the discrepancy. Several integrin subunits, including $\alpha_{v}$, are recycled through the endocytic pathway, whereas activated $\alpha_{2} \beta_{1}$ induces caveolae-mediated endocytosis [Upla et al., 2004]. These pathways may be differentially regulated in modeled microgravity. Another plausible explanation for differential regulation of integrin subunits is ligand availability. The $\alpha_{v} \beta_{3}$ receptor binds vitronectin and other RGD-containing peptides [Wong et al., 1996]. In contrast to the severe reduction in Col I expression we have observed, spaceflight studies have revealed no change in mRNA expression, protein expression, or matrix accumulation of the RGDcontaining extracellular matrix protein, fibronectin [Hughes-Fulford and Gilbertson, 1999]. Therefore, it is possible that the difference in ligand availability contributes to the differential regulation of distinct integrin subunits.

To address the effects of reduced Col $\mathrm{I}$ and altered integrin subunit expression on integrin function, we analyzed the autophosphorylation of two adhesion-dependent tyrosine kinases, FAK and PYK2. We observed a significant reduction in FAK and PYK2 autophosphorylation in hMSC after 7 days culture in modeled microgravity. This is consistent with integrin regulation of osteoblastic differentiation. Activation of FAK [Freitas et al., 2002] and PYK2 [Jeschke et al., 1998] has been linked to the anabolic action of fluoride treatment in the embryonic mouse MC3T3-E1 cell line. Mechanical strain has also been shown to enhance FAK and PYK2 autophosphorylation in ROS $17 / 2.8$ osteoblast-like cells [Boutahar et al., 2004]. Given the reduced mechanical load on our cells in modeled microgravity, the reduction in FAK and PYK2 activation are consistent with these data. The role of FAK alone in osteoblastic differentiation has been studied more extensively. Transfection of MC3T3-E1 cells with antisense FAK leads to suppressed alkaline phosphatase activity and osteocalcin gene 
expression in response to BMP-2 treatment [Tamura et al., 2001]. In addition, disruption of focal contacts by cytochalasin D blocks Col l-stimulated increases in alkaline phosphatase activity [Takeuchi et al., 1997]. Interestingly, osteoblasts isolated from patients suffering from osteoporosis or osteoarthritis exhibit reduced attachment and spreading, less defined focal adhesions and stress fibers, and reduced FAK phosphorylation [Perinpanayagam et al., 2001]. FAK activation is therefore important for osteoblastic differentiation and likely contributes to the reduced differentiation we observed previously [Zayzafoon et al., 2004].

Due to the observed reduction in FAK autophosphorylation, we anticipated a concomitant reduction in activation of the Ras-MAPK pathway. As expected, we detected a significant decline in Ras and ERK activation in hMSC cultured in modeled microgravity. These results are supported by a study in which overexpression of antisense FAK in MC3T3-E1 cells, prevents collagen-induced Ras-MAPK activation [Takeuchi et al., 1997]. Conversely, overexpression of FAK in human 293 epithelial cells leads to enhanced Ras-dependent activation of ERK [Schlaepfer and Hunter, 1997]. Interestingly, centrifugation-induced hypergravity leads to an induction of ERK phosphorylation in human osteoblastic cells [Gebken et al., 1999]. Sustained activation of ERK is required for activation of Runx2, an essential transcription factor for osteoblastic differentiation [Xiao et al., 2000]. It is therefore likely that reduced ERK activation in modeled microgravity contributes to the subsequent reduction in osteoblastic differentiation of hMSC.

Autophosphorylation of FAK has also been implicated in direct activation of PI3K [Chen et al., 1996], and overexpression of FAK leads to a PI3K-dependent increase in cell proliferation [Yamamoto et al., 2003]. Despite a reduction in FAK autophosphorylation, we detected no change in the activation of Akt, a downstream target of PI3K. Unaltered activation of Akt is consistent with our previous data showing no change in hMSC proliferation during culture in modeled microgravity compared to normal gravity [Zayzafoon et al., 2004]. One interesting candidate for maintenance of PI3KJAkt activation in our system is leptin. We have previously 
demonstrated an increase in leptin expression by hMSC cultured for 7 days in modeled microgravity [Zayzafoon et al., 2004]. Leptin has been shown to activate PI3K in human peripheral blood mononuclear cells [Martin-Romero and Sanchez-Margalet, 2001], and leptin administration in vivo leads to a significant increase in Akt phosphorylation [Maroni et al., 2003]. It is also likely that growth factors and cytokines present in the serum contribute to PI3K/Akt activation as well.

This work provides new insights into potential mechanisms leading to reduced bone mass in humans during spaceflight. Using the RCCS, we show that 7 days culture in modeled microgravity inhibits osteoblastic gene expression in hMSC, including expression of Col 1 . Despite upregulation of the Col-l specific integrin subunits, $\alpha_{2}$ and $\beta_{1}$, integrin signaling through FAK, Ras, and ERK is significantly reduced and likely contributes to reduced osteoblastic differentiation. It may be possible, then, to develop anabolic bone therapeutic agents aimed at triggering integrin signaling to prevent bone loss associated with spaceflight, disuse, and aging osteopenia. 


\section{REFERENCES}

Bikle DD, Halloran BP (1999): The response of bone to unloading. J Bone Miner Metab 17:23344.

Boutahar N, Guignandon A, Vico L, Lafage-Proust MH (2004): Mechanical strain on osteoblasts activates autophosphorylation of FAK and PYK2 tyrosine sites involved in ERK activation. J Biol Chem.

Caillot-Augusseau A, Vico L, Heer M, Voroviev D, Souberbielle JC, Zitterman A, Alexandre C, Lafage-Proust $\mathrm{MH}$ (2000): Space flight is associated with rapid decreases of undercarboxylated osteocalcin and increases of markers of bone resorption without changes in their circadian variation: observations in two cosmonauts. Clin Chem 46:1136-43.

Carmeliet G, Nys G, Stockmans I, Bouillon R (1998): Gene expression related to the differentiation of osteoblastic cells is altered by microgravity. Bone 22:139S-143S.

Chen HC, Appeddu PA, Isoda H, Guan JL (1996): Phosphorylation of tyrosine 397 in focal adhesion kinase is required for binding phosphatidylinositol 3-kinase. J Biol Chem 271:2632934.

Collet P, Uebelhart D, Vico L, Moro L, Hartmann D, Roth M, Alexandre C (1997): Effects of 1and 6-month spaceflight on bone mass and biochemistry in two humans. Bone 20:547-51. Freitas F, Jeschke M, Majstorovic 1, Mueller DR, Schindler P, Voshol H, Van Oostrum J, Susa M (2002): Fluoroaluminate stimulates phosphorylation of p130 Cas and Fak and increases attachment and spreading of preosteoblastic MC3T3-E1 cells. Bone 30:99-108.

Gebken J, Luders B, Notbohm H, Klein HH, Brinckmann J, Muller PK, Batge B (1999): Hypergravity stimulates collagen synthesis in human osteoblast-like cells: evidence for the involvement of p44/42 MAP-kinases (ERK 1/2). J Biochem (Tokyo) 126:676-82.

Ghosh-Choudhury N, Abboud SL, Nishimura R, Celeste A, Mahimainathan L, Choudhury GG (2002): Requirement of BMP-2-induced Phosphatidylinositol 3-Kinase and Akt Serine/Threonine Kinase in Osteoblast Differentiation and Smad-dependent BMP-2 Gene Transcription. J. Biol. Chem. 277:33361-33368.

Gronthos S, Simmons PJ, Graves SE, Robey PG (2001): Integrin-mediated interactions between human bone marrow stromal precursor cells and the extracellular matrix. Bone 28:17481.

Hanks SK, Ryzhova L, Shin NY, Brabek J (2003): Focal adhesion kinase signaling activities and their implications in the control of cell survival and motility. Front Biosci 8:d982-96.

Hughes-Fulford M, Gilbertson V (1999): Osteoblast fibronectin mRNA, protein synthesis, and matrix are unchanged after exposure to microgravity. FASEB J. 13:121-127.

Hynes RO (2002): Integrins: bidirectional, allosteric signaling machines. Cell 110:673-87.

Jeschke M, Standke GJ, Scaronuscarona M (1998): Fluoroaluminate induces activation and association of Src and Pyk2 tyrosine kinases in osteoblastic MC3T3-E1 cells. J Biol Chem 273:11354-61.

Juliano RL (2002): Signal transduction by cell adhesion receptors and the cytoskeleton: functions of integrins, cadherins, selectins, and immunoglobulin-superfamily members. Annu Rev Pharmacol Toxicol 42:283-323.

Kafantari H, Kounadi E, Fatouros M, Milonakis M, Tzaphlidou M (2000): Structural alterations in rat skin and bone collagen fibrils induced by ovariectomy. Bone 26:349-353.

Lai CF, Chaudhary L, Fausto A, Halstead LR, Ory DS, Avioli LV, Cheng SL (2001): Erk is essential for growth, differentiation, integrin expression, and cell function in human osteoblastic cells. J Biol Chem 276:14443-50.

Landis WJ, Hodgens KJ, Block D, Toma CD, Gerstenfeld LC (2000): Spaceflight effects on cultured embryonic chick bone cells. J Bone Miner Res 15:1099-112. 
Maroni P, Bendinelli P, Piccoletti R (2003): Early intracellular events induced by in vivo leptin treatment in mouse skeletal muscle. Molecular and Cellular Endocrinology 201:109-121. Martin-Romero C, Sanchez-Margalet V (2001): Human Leptin Activates PI3K and MAPK Pathways in Human Peripheral Blood Mononuclear Cells: Possible Role of Sam68. Cellular Immunology 212:83-91.

Moore S, Spackman DH, Stein WH (1958): Automatic recording apparatus for use in the chromatography of amino acids. Fed Proc 17:1107-15.

Nakamura H, Kumei Y, Morita S, Shimokawa H, Ohya K, Shinomiya K (2003): Suppression of osteoblastic phenotypes and modulation of pro- and anti-apoptotic features in normal human osteoblastic cells under a vector-averaged gravity condition. J Med Dent Sci 50:167-76. Narayanan R, Smith CL, Weigel NL (2002): Vector-averaged gravity-induced changes in cell signaling and vitamin D receptor activity in MG-63 cells are reversed by a 1,25-(OH)(2)D(3) analog, EB1089. Bone 31:381-8.

Owen TA, Aronow M, Shalhoub V, Barone LM, Wilming L, Tassinari MS, Kennedy MB, Pockwinse S, Lian JB, Stein GS (1990): Progressive development of the rat osteoblast phenotype in vitro: reciprocal relationships in expression of genes associated with osteoblast proliferation and differentiation during formation of the bone extracellular matrix. J Cell Physiol 143:420-30.

Perinpanayagam H, Zaharias R, Stanford C, Brand R, Keller J, Schneider G (2001): Early cell adhesion events differ between osteoporotic and non-osteoporotic osteoblasts. J Orthop Res 19:993-1000.

Pittenger MF, Mackay AM, Beck SC, Jaiswal RK, Douglas R, Mosca JD, Moorman MA, Simonetti DW, Craig S, Marshak DR (1999): Multilineage potential of adult human mesenchymal stem cells. Science 284:143-7.

Rodriguez JP, Montecinos L, Rios S, Reyes P, Martinez J (2000): Mesenchymal stem cells from osteoporotic patients produce a type I collagen-deficient extracellular matrix favoring adipogenic differentiation. J Cell Biochem 79:557-65.

Sakata T, Wang Y, Halioran BP, Elalieh HZ, Cao J, Bikle DD (2004): Skeletal Unloading Induces Resistance to Insulin-Like Growth Factor-I (IGF-I) by Inhibiting Activation of the IGF-I Signaling Pathways. J Bone Miner Res 19:436-446.

Schlaepfer DD, Hunter T (1997): Focal Adhesion Kinase Overexpression Enhances Rasdependent Integrin Signaling to ERK2/Mitogen-activated Protein Kinase through Interactions with and Activation of C-Src. J. Biol. Chem. 272:13189-13195.

Schneider GB, Zaharias R, Stanford C (2001): Osteoblast integrin adhesion and signaling regulate mineralization. J Dent Res 80:1540-4.

Siggelkow H, Rebenstorff K, Kurre W, Niedhart C, Engel I, Schulz H, Atkinson MJ, Hufner M (1999): Development of the osteoblast phenotype in primary human osteoblasts in culture: comparison with rat calvarial cells in osteoblast differentiation. J Cell Biochem 75:22-35.

Suzawa M, Tamura Y, Fukumoto S, Miyazono K, Fujita T, Kato S, Takeuchi Y (2002):

Stimulation of Smad1 transcriptional activity by Ras-extracellular signal-regulated kinase pathway: a possible mechanism for collagen-dependent osteoblastic differentiation. J Bone Miner Res 17:240-8.

Takeuchi Y, Suzawa M, Kikuchi T, Nishida E, Fujita T, Matsumoto T (1997): Differentiation and transforming growth factor-beta receptor down-regulation by collagen-alpha2beta1 integrin interaction is mediated by focal adhesion kinase and its downstream signals in murine osteoblastic cells. J Biol Chem 272:29309-16.

Tamura Y, Takeuchi Y, Suzawa M, Fukumoto S, Kato M, Miyazono K, Fujita T (2001): Focal adhesion kinase activity is required for bone morphogenetic protein--Smad1 signaling and osteoblastic differentiation in murine MC3T3-E1 cells. J Bone Miner Res 16:1772-9. Tilton FE, Degioanni JJ, Schneider VS (1980): Long-term follow-up of Skylab bone demineralization. Aviat Space Environ Med 51:1209-13. 
Upla P, Marjomaki V, Kankaanpaa P, Ivaska J, Hyypia T, van der Goot FG, Heino J (2004):

Clustering Induces a Lateral Redistribution of \{alpha\}2\{beta\}1 Integrin from Membrane Rafts to Caveolae and Subsequent Protein Kinase C-dependent Internalization. Mol. Biol. Cell 15:625636.

Vinals F, Lopez-Rovira T, Rosa JL, Ventura F (2002): Inhibition of PI3K/p70 S6K and p38 MAPK cascades increases osteoblastic differentiation induced by BMP-2. FEBS Lett 510:99104.

Wong A, Hwang S, McDevitt P, McNulty D, Stadel J, Johanson K (1996): Studies on alpha v beta 3/ligand interactions using a [3H]SK\&F-107260 binding assay. Mol Pharmacol 50:529-537.

Xiao G, Cui Y, Ducy P, Karsenty G, Franceschi RT (1997): Ascorbic Acid-Dependent Activation of the Osteocalcin Promoter in MC3T3-E1 Preosteoblasts: Requirement for Collagen Matrix Synthesis and the Presence of an Intact OSE2 Sequence. Mol Endocrinol 11:1103-1113.

Xiao G, Gopalakrishnan R, Jiang D, Reith E, Benson MD, Franceschi RT (2002): Bone morphogenetic proteins, extracellular matrix, and mitogen-activated protein kinase signaling pathways are required for osteoblast-specific gene expression and differentiation in MC3T3-E1 cells. J Bone Miner Res 17:101-10.

Xiao G, Jiang D, Thomas P, Benson MD, Guan K, Karsenty G, Franceschi RT (2000): MAPK Pathways Activate and Phosphorylate the Osteoblast-specific Transcription Factor, Cbfa1. J. Biol. Chem. 275:4453-4459.

Xiao G, Wang D, Benson MD, Karsenty G, Franceschi RT (1998): Role of the alpha2-integrin in osteoblast-specific gene expression and activation of the Osf2 transcription factor. J Biol Chem 273:32988-94.

Xiong WC, Feng X (2003): PYK2 and FAK in osteoclasts. Front Biosci 8:d1219-26.

Yamamoto D, Sonoda Y, Hasegawa M, Funakoshi-Tago M, Aizu-Yokota E, Kasahara T (2003): FAK overexpression upregulates cyclin D3 and enhances cell proliferation via the PKC and PI3kinase-Akt pathways. Cellular Signalling 15:575-583.

Zayzafoon M, Gathings WE, McDonald JM (2004): Modeled Microgravity Inhibits Osteogenic Differentiation of Human Mesenchymal Stem Cells and Increases Adipogenesis. Endocrinology 145:2421-2432. 
Figure 1. Modeled microgravity reduces osteoblastic gene expression. hMSC were seeded onto plastic microcarrier beads and cultured for 7 days in DMEM $+10 \%$ FBS under normal gravity conditions. Aggregates were then maintained in osteogenic medium for 7 days in normal gravity or modeled microgravity. Total RNA was extracted at 0,7 , and 14 days, and semi-quantitative RT-PCR reactions were performed using primers for alkaline phosphatase (ALP) and Runx2. Relative gene expression (mean \pm SEM) from 4 separate experiments was normalized to $18 \mathrm{~S}$ expression and plotted on a log scale.

Figure 2. Collagen I expression is reduced in modeled microgravity. hMSC were seeded onto plastic microcarrier beads and cultured for 7 days in DMEM $+10 \%$ FBS under normal gravity conditions. Aggregates were then maintained in osteogenic medium for 7 days in normal gravity or modeled microgravity. A) Total RNA was extracted at days 0,7 , and 14, and semiquantitative RT-PCR reactions were performed using primers to type I procollagen (Col I $\alpha 2$ ). Relative gene expression (mean \pm SEM) from 4 separate experiments was normalized to $18 \mathrm{~S}$ expression and plotted on a log scale. B) Whole aggregates, harvested at day 14 , were used for hydroxyproline analysis. Values were obtained from 2 separate experiments in duplicate and represent the average hydroxyproline content per aggregate.

Figure 3. Integrin subunit protein expression is altered in modeled microgravity. hMSC were seeded onto plastic microcarrier beads and cultured for 7 days in proliferation medium. Aggregates were then maintained in osteogenic medium for 7 days at either unit gravity (G) or modeled microgravity (MMG). Total protein was extracted at the end of the study, and extracts (20 $\mu \mathrm{g} /$ lane) were separated by $7.5 \%$ SDS-PAGE. A) Immunoblots were developed using specific antibodies to $\beta 1$ or $\alpha 2$ integrin subunits. Images are representative of at least 3 separate experiments. B) Levels of integrin subunit expression in MMG are graphed relative to 
gravity controls (G). Values were obtained from at least 3 separate experiments and represent the mean \pm SE. ${ }^{*} p<0.05$.

Figure 4. Modeled microgravity decreases autophosphorylation of FAK and PYK2. hMSC were seeded onto plastic microcarrier beads and cultured for 7 days in proliferation medium. Aggregates were then maintained in osteogenic medium for 7 days at either unit gravity $(G)$ or modeled microgravity (MMG). Total protein was extracted at the end of the study, and extracts (20 $\mu \mathrm{g} / \mathrm{lane}$ ) were separated by $7.5 \%$ SDS-PAGE. A) Immunoblots were developed using specific antibodies to phosphorylated tyrosine residue 397 on FAK or total FAK. Images are representative of 4 separate experiments. B) Levels of FAK autophosphorylation in MMG are expressed relative to total FAK and graphed as a percentage relative to gravity controls (G). Values were obtained from 4 separate experiments and represent the mean \pm SE. ${ }^{*} p<0.001$.

Figure 5. Ras activity and ERK phosphorylation are reduced in modeled microgravity. hMSC were seeded onto plastic microcarrier beads and cultured for 7 days in proliferation medium. Aggregates were then maintained in osteogenic medium for 7 days at either unit gravity (G) or modeled microgravity (MMG). Total protein was extracted at the end of the study. A) Activated (GTP-bound) Ras was pulled down from total protein $(150 \mu \mathrm{g})$ using Raf-1 conjugated agarose beads and separated by $12.5 \%$ SDS-PAGE. Immunoblots were developed using specific antibodies to Ras. The image is representative of 3 separate experiments. Levels of GTP-Ras in MMG are expressed as a percentage relative to gravity controls (G). Values were obtained from 3 separate experiments and represent the mean $+S E{ }^{*} p<0.05$. B) Protein extracts were separated by $10 \%$ SDS-PAGE. Immunoblots were developed using specific antibodies to phosphorylated or total ERK. The image is representative of 6 separate experiments. Levels of ERK phosphorylation in MMG are expressed relative to total ERK and graphed as a percentage 
relative to gravity controls $(G)$. Values were obtained from 6 separate experiments and represent the mean $\pm S . E .{ }^{*} p<0.05$

Figure 6. Modeled microgravity does not alter activation of Akt. hMSC were seeded onto plastic microcarrier beads and cultured for 7 days in proliferation medium. Aggregates were then maintained in osteogenic medium for 7 days at either unit gravity (G) or modeled microgravity (MMG). Total protein was extracted at the end of the study and separated by $10 \%$ SDS-PAGE. Immunoblots were developed using specific antibodies to phosphorylated or total Akt. The image is representative of 2 separate experiments.

Figure 7. Potential regulation of osteoblastic differentiation through reduced integrin signaling in modeled microgravity. Modeled microgravity suppresses expression and matrix accumulation of Col 1. Expression of Col l-specific integrin subunits $\alpha_{2}$ and $\beta_{1}$, is significantly increased in modeled microgravity. However, reduced Col 1 availability results in reduced downstream signaling through adhesion-dependent kinases FAK and PYK2. There is a subsequent reduction in Ras and ERK activation, which contributes to reduced Runx2 activation. This results in diminished transcription of osteoblastic genes, including type I collagen. 
Figure 1.

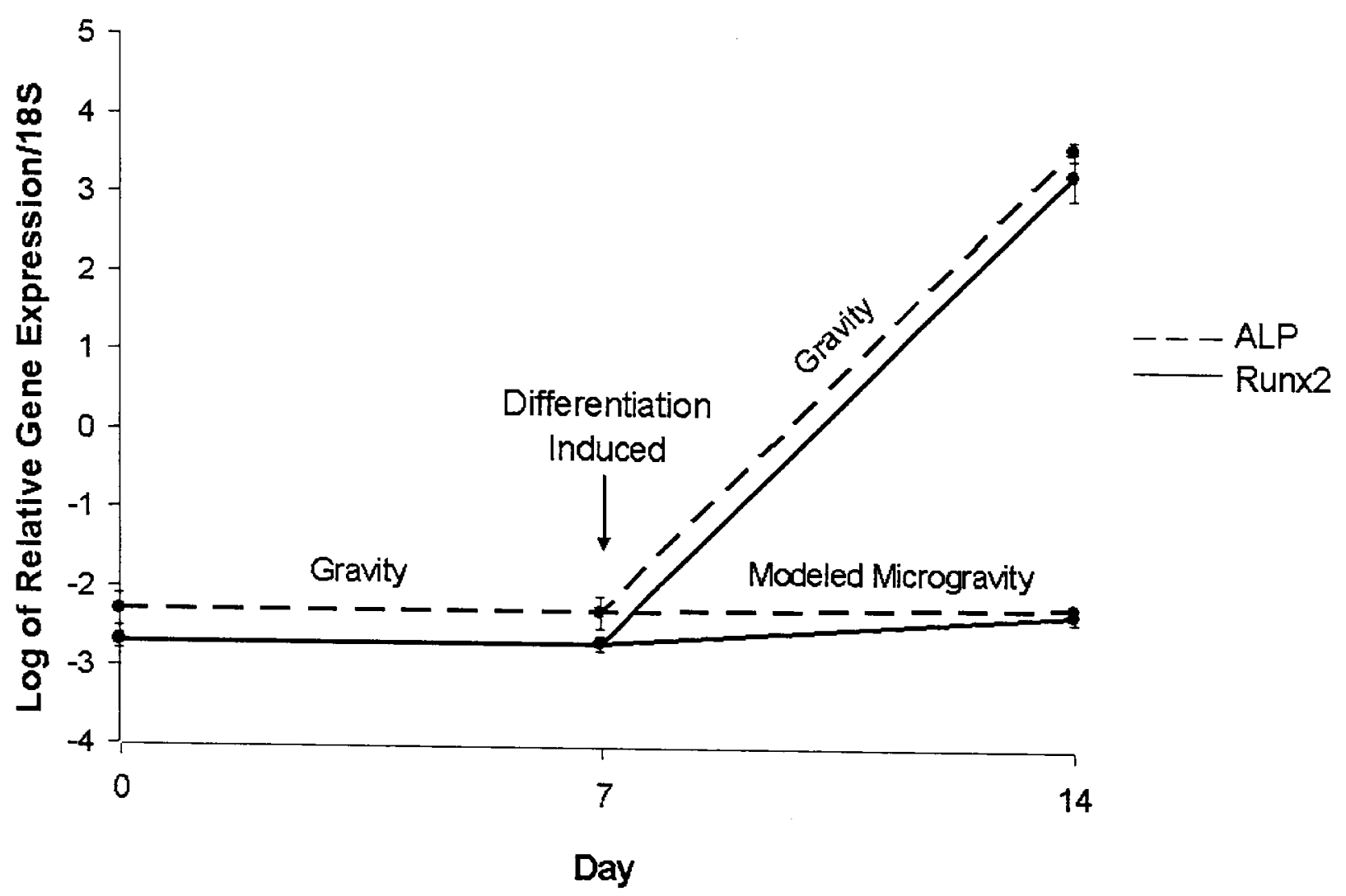


Figure 2.

A)

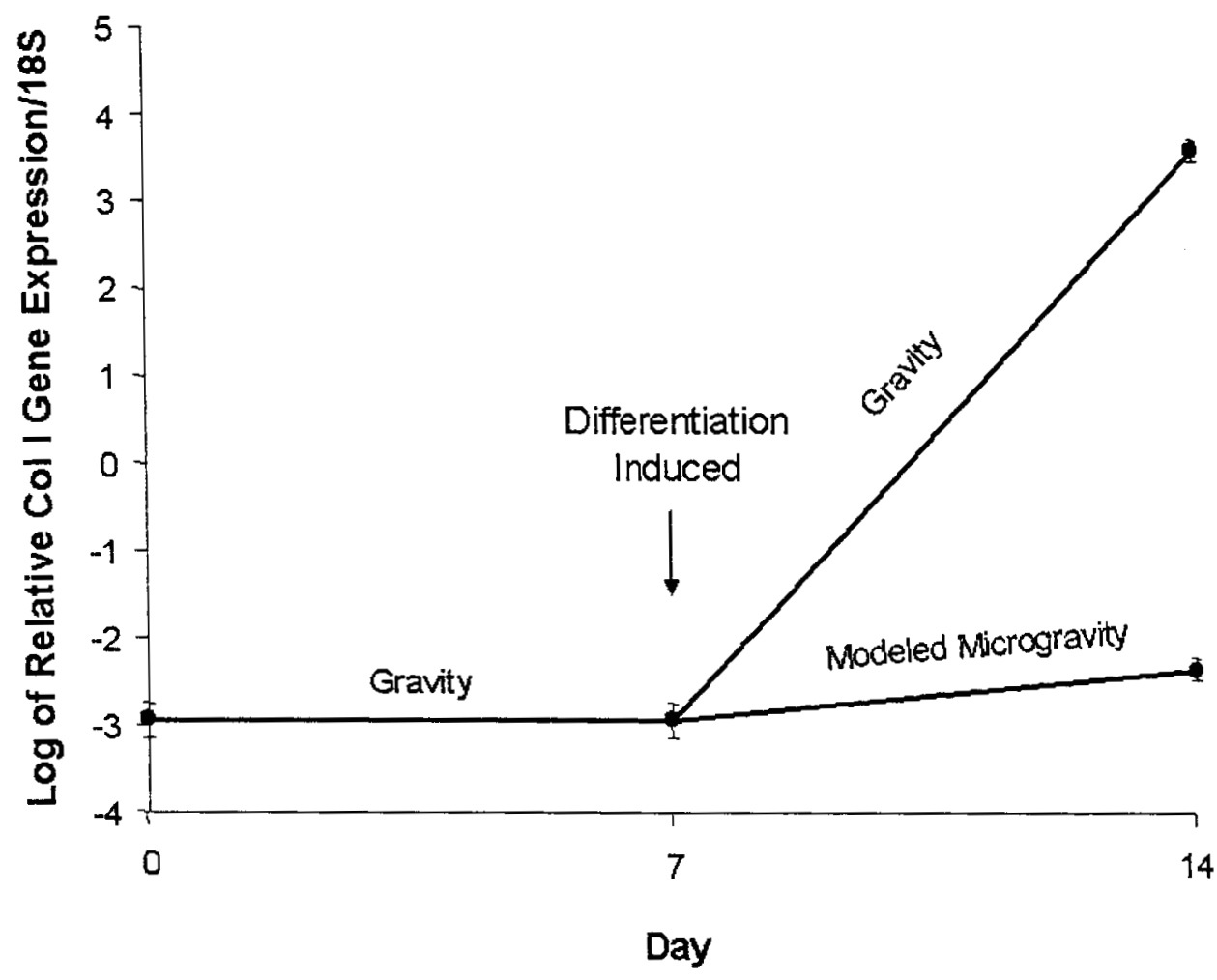

B)

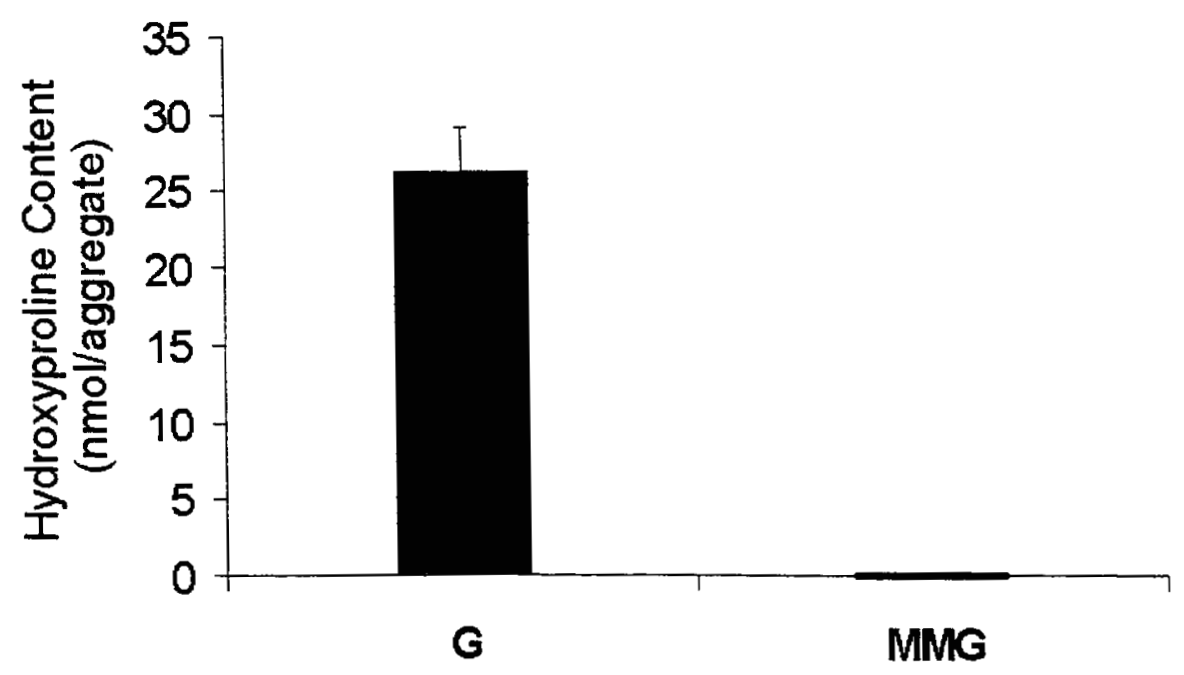


Figure 3.

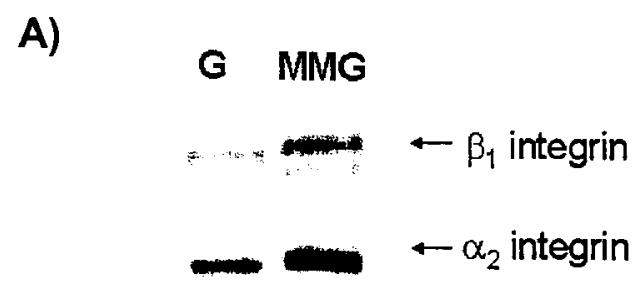

B)

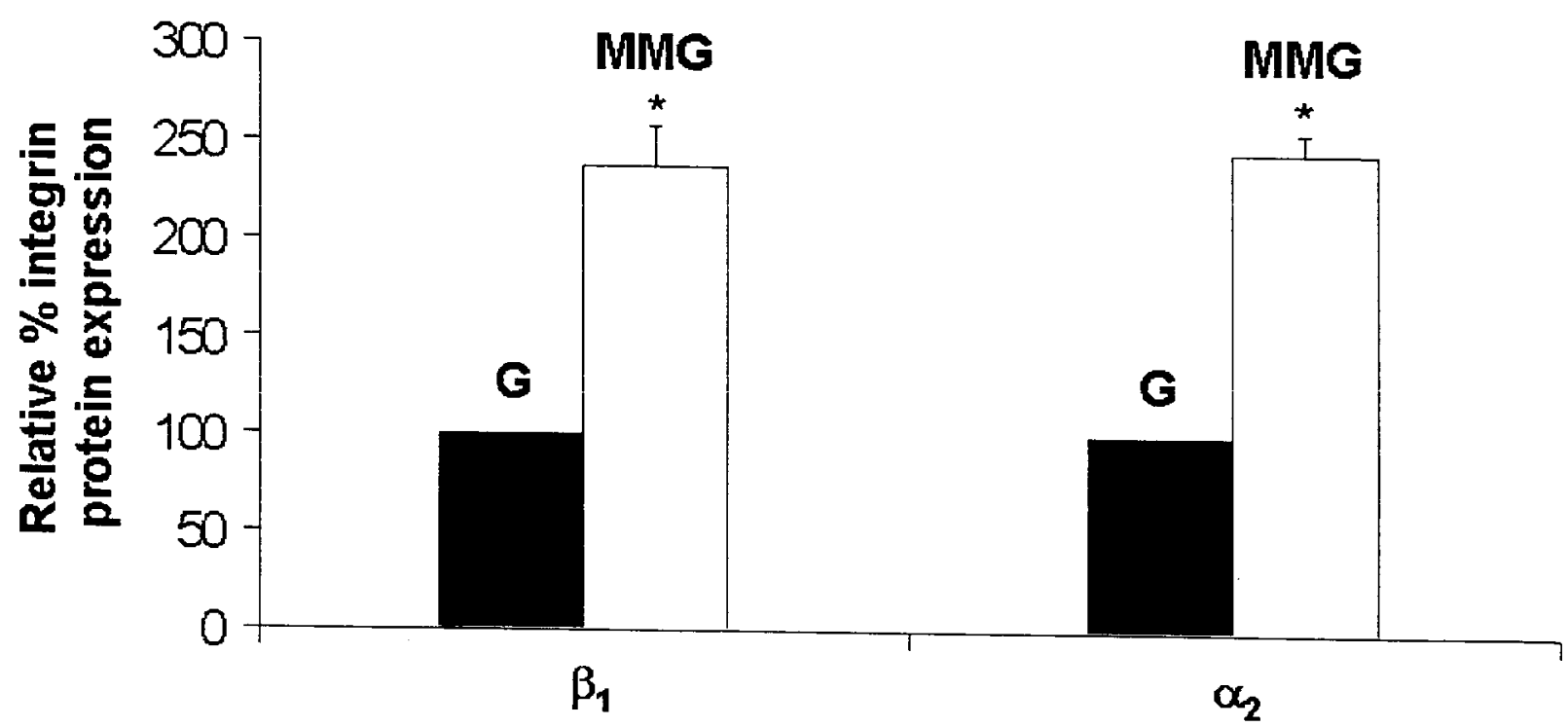


Figure 4.

A)

\begin{tabular}{|c|c|c|c|c|}
\hline $\mathbf{G}$ & MMG & G & MMG & \\
\hline m & $\leftarrow p-397 \mathrm{FAK}$ & 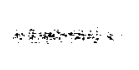 & & $\leftarrow p-402$ PYK2 \\
\hline
\end{tabular}

B)

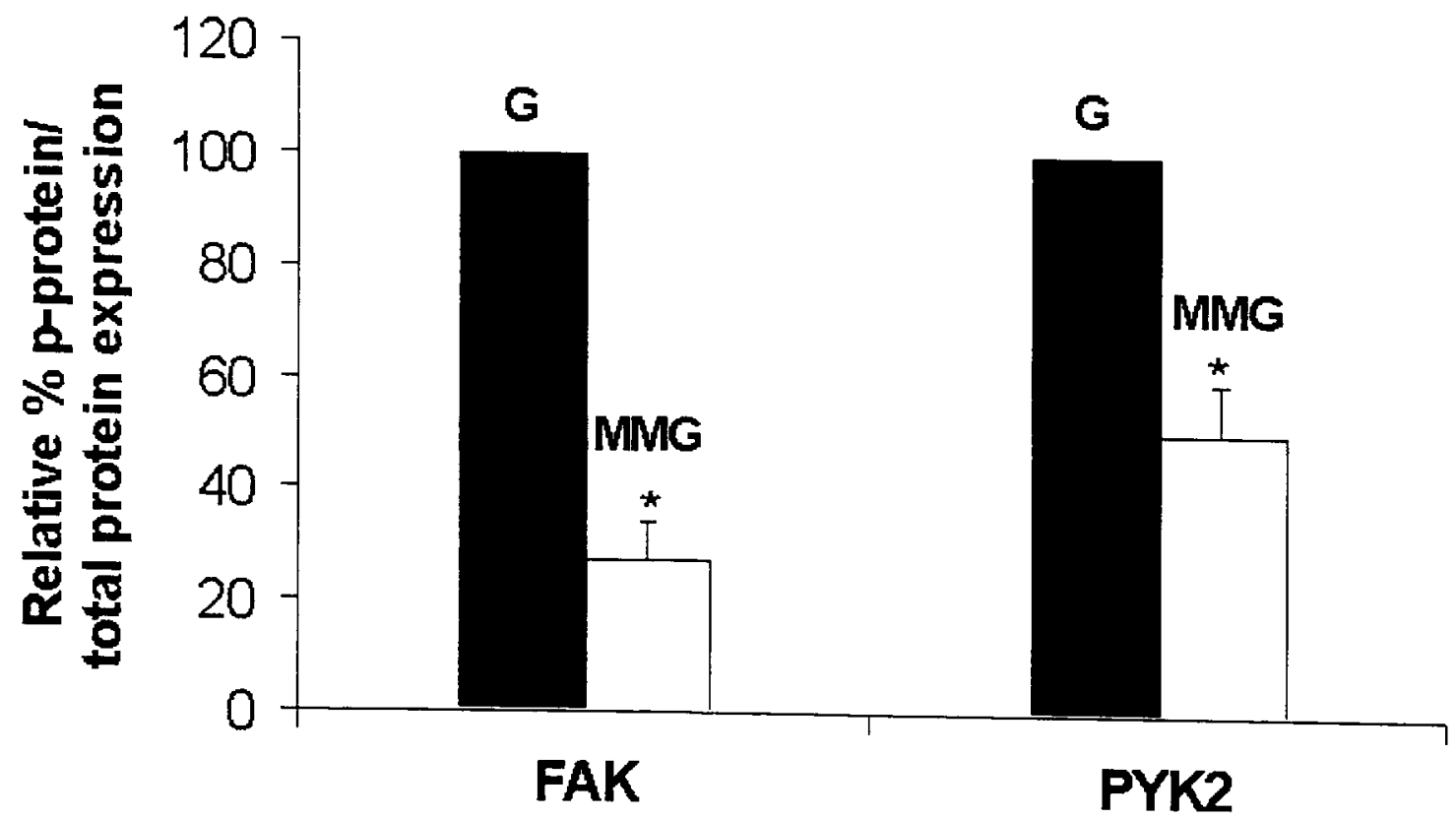


Figure 5.

A)

$$
\begin{array}{llll}
-\quad \text { M MMG } & \\
\text { GTP-Ras } & &
\end{array}
$$

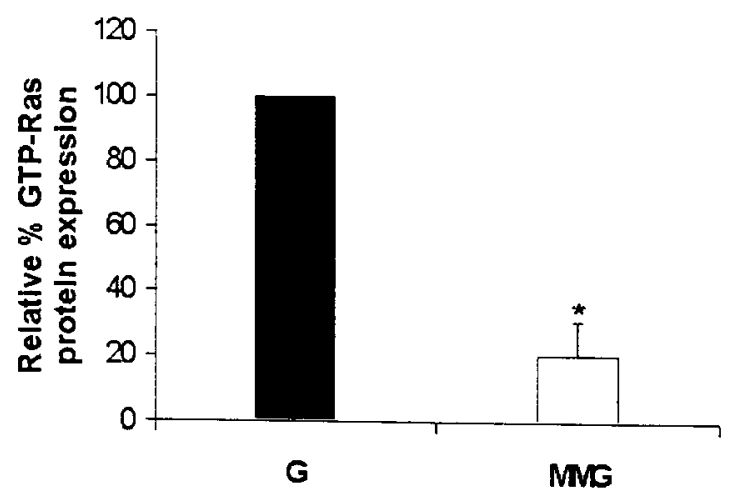

B)
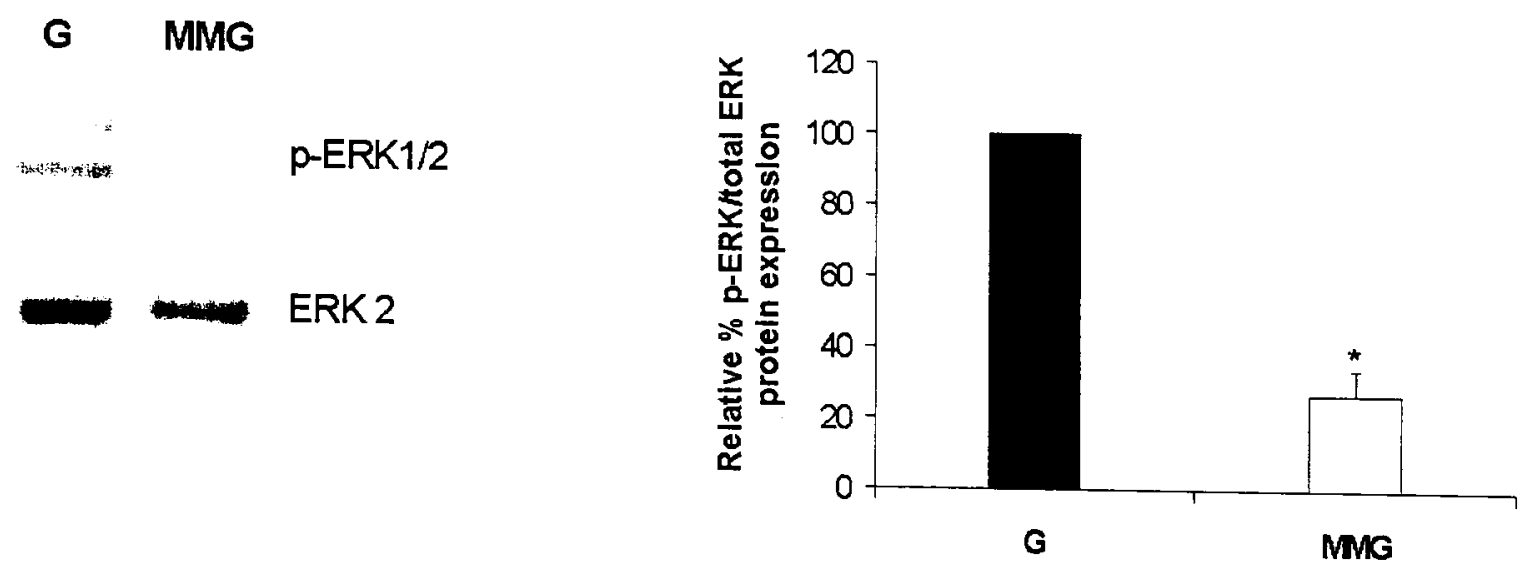
Figure 6.

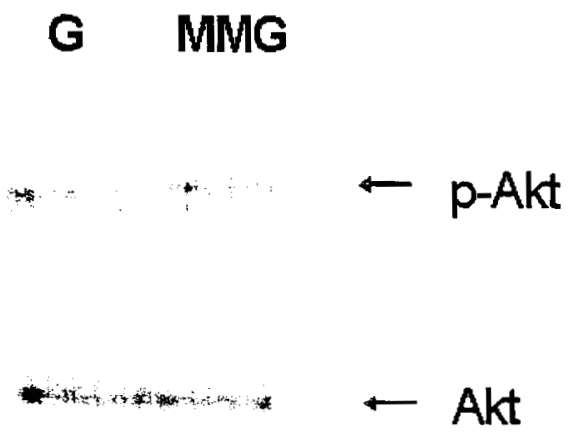


Figure 7.

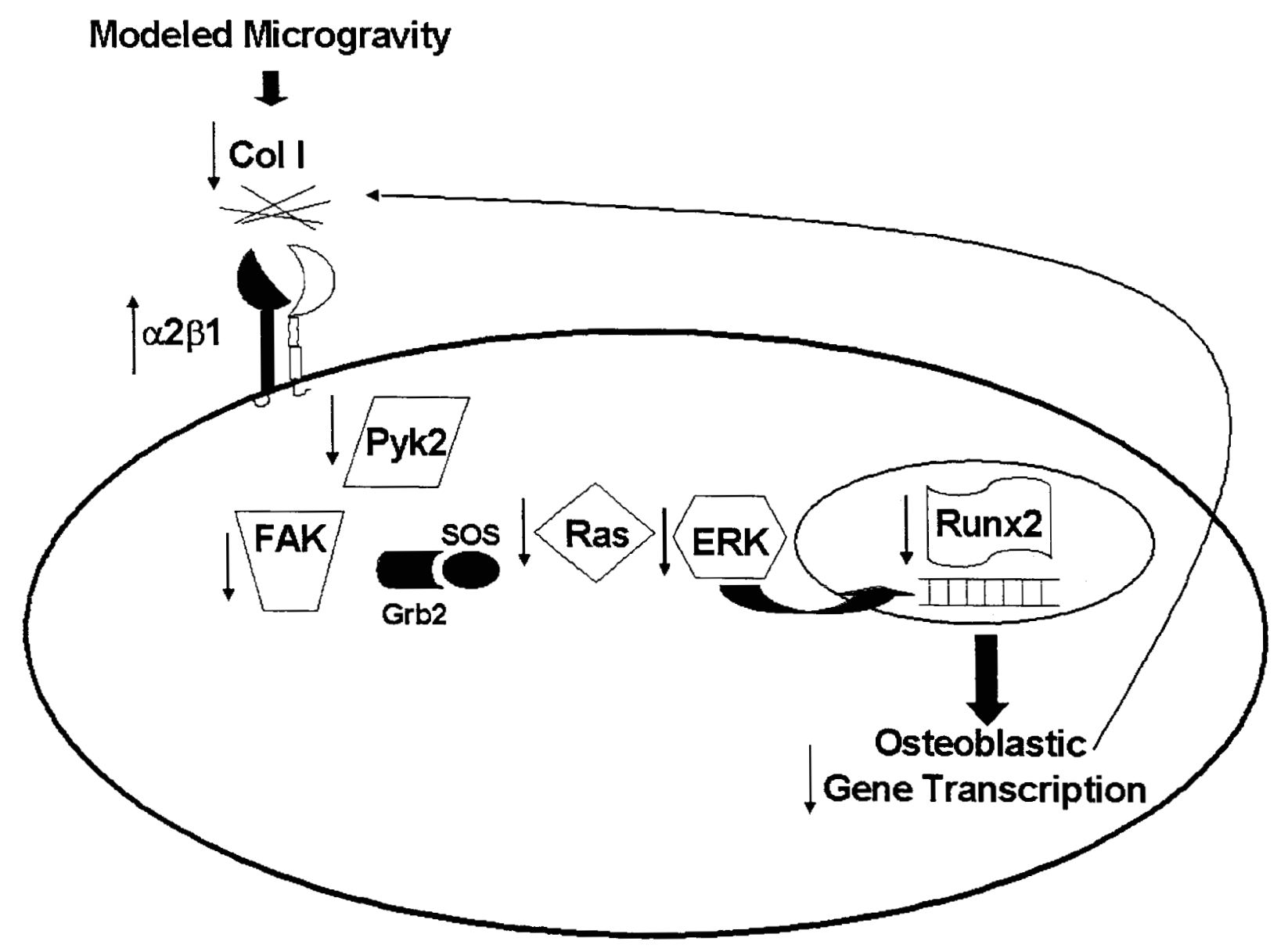

